\section{Terapia de reposição hormonal e o câncer do endométrio}

\author{
Hormone replacement therapy and \\ endometrial cancer
}

\section{${ }^{1}$ Escola Bahiana de Medicina e Saúde Pública, Salvador, Brasil. \\ 2 Centro de Pesquisa Gonçalo Moniz, Fundação Oswaldo Cruz, Salvador, Brasil. \\ Correspondência D. A. Athanazio Centro de Pesquisa Gonçalo Moniz, Fundação Oswaldo Cruz. \\ Rua Waldemar Falcão 121, Salvador, $B A$ \\ 40296-710, Brasil. \\ dathanazio@cpqgm.fiocruz.br}

\begin{abstract}
Hormone replacement therapy (HRT) has been used worldwide to relieve menopausal symptoms. The evolution in clinical knowledge of its risks has lagged persistently behind its application in clinical practice. In the 1970s, endometrial cancer incidence increased in developed countries, and exogenous estrogen therapy in postmenopausal women was the most important factor. In the 1980s and 90s, combined estrogen-progesterone therapy was prescribed on a large scale, based on its potential beneficial effects on the musculoskeletal and cardiovascular systems without increasing the risk of endometrial carcinoma. However, once again, recent reports argue against the safety of HRT, and the most important issues are now the increased risk of cardiovascular disease and total cancer in combined formulations. We conclude that the recently reported risks on combined HRT may have a significant public health impact, and that this treatment regimen should not be prescribed to protect postmenopausal women from endometrial carcinoma, since the risks outweigh the benefits.
\end{abstract}

Hormone Replacement Therapy; Endometrial Neoplasms; Women's Health
Naidilton Lantyer Cordeiro de Araújo Júnior 1 Daniel Abensur Athanazio 1,2

\section{Introdução}

A aplicação clínica da terapia de reposição hormonal (TRH) é um bom exemplo de como a evolução do conhecimento médico pode ser tortuosa, e como a comunidade médica e científica pode subestimar por considerável período os efeitos de algumas intervenções. O uso de hormônios para aliviar a sintomatologia relacionada à menopausa é amplamente praticado em todo mundo, e potenciais efeitos adversos são de grande interesse para Saúde Pública devido ao potencial impacto numa grande população exposta.

Nas últimas três décadas, a principal questão relacionada à TRH foi o risco de câncer do endométrio. $\mathrm{O}$ câncer endometrial é a sétima neoplasia maligna mais comum do mundo sendo dez vezes mais incidente em países desenvolvidos. O aumento na incidência da doença parece estar relacionado à epidemia de obesidade e à crescente expectativa de vida nas nações mais ricas. Cerca de $80 \%$ de todos os casos são adenocarcinomas de padrão endometrióide cuja relação com hiperplasia endometrial prévia é bem estabelecida. Os principais fatores de risco relacionados à hiperplasia endometrial e progressão para o câncer estão relacionados à hiperestimulação do endométrio pela exposição crônica aos altos níveis circulantes de estrógenos, sem oposição de efeitos antiproliferativos da progesterona. Estes incluem obesidade, dieta hiperlipídica e 
sedentarismo sendo que a conversão periférica no tecido adiposo de androgênios em estrógenos parece ser o principal mecanismo relacionado. A história reprodutiva é também importante visto que mulheres nuligestas, que não sofreram efeitos de longos períodos de produção de progesterona pela placenta, também têm risco elevado. Como será discutido em detalhe adiante, o uso de estrógenos exógenos sem oposição de progestínicos é também um importante fator de risco para o câncer de endométrio. O prognóstico da doença é determinado principalmente pelo seu estadiamento e a sobrevida em cinco anos é estimada em $85 \%$ para o estágio I (restrito ao corpo uterino), $75 \%$ para o estágio II (extensão para o colo), 45\% para o estágio III (extensão para outras estruturas pélvicas) e 25\% para o estágio IV (invasão da mucosa vesical ou colônica, ou metástases extra-pélvicas) 1 .

Neste trabalho de revisão, trataremos da evolução sobre o conhecimento dos efeitos da TRH sobre o endométrio e discutiremos os recentes relatos que novamente questionam a segurança de seu uso, desta vez não só com relação ao câncer de endométrio, mas também associando-a com o câncer de mama e complicações cardiovasculares.

\section{Esquemas usados para reposição hormonal}

Estrógenos podem ser divididos em naturais e sintéticos. Os naturais são mais utilizados na reposição hormonal, enquanto os sintéticos são aplicados em métodos de contracepção. Os hormônios naturais são compostos basicamente por estradiol, estrona e estriol. Os dois primeiros são produtos de síntese ovariana, enquanto o terceiro é conseqüência do metabolismo periférico do estradiol e estrona. O potencial biológico dos diferentes tipos de estrógenos depende basicamente da afinidade de cada tipo de molécula ao seu receptor-alvo. Demonstrou-se que o tempo de retenção do estradiol no receptor-alvo é maior que o da estrona sendo, portanto, um hormônio mais potente. $\mathrm{O}$ estriol é um estrógeno fraco e sua afinidade com o receptor é de $20 \%$ a $30 \%$ quando comparado com a molécula de estradiol.

O metabolismo dos estrógenos ocorre principalmente no fígado onde a estrona é transformada em estriol e epiestriol. Há também a conjugação de tais metabólitos em sulfatos e glicuronatos, que são altamente hidrossolúveis e facilmente excretados pelos rins. Metade dos estrógenos é excretada na urina nas primeiras 24 horas e outra metade é inicialmente eliminada pela bile sofrendo hidrólise no intestino onde até $80 \%$ são reabsorvidos. Ao retornar ao fígado, podem escapar da reconjugação e entrar na corrente sanguínea ou podem ser reconjugados e excretados na bile ou urina. Somente $10 \%$ dos estrógenos são eliminados nas fezes.

Os estrógenos conjugados são hormônios naturais extraídos da urina de éguas prenhes e incluem, além de estradiol, estrona, sulfato de estrona e os estrógenos derivados do anel B insaturado: equilina, equilenina e seus sulfatos. Os efeitos biológicos dos estrógenos conjugados são resultantes principalmente da combinação de sulfato de estrona, sulfato de equilina e seus metabólitos. A equilina, por si só, é mais potente que todos os outros componentes dos estrógenos conjugados.

As vias de administração de estrógenos naturais são a oral, transdérmica, percutânea e vaginal. Pela via oral, os estrógenos passam pelo fígado e são metabolizados em estrona e sulfato de estrona. As vias transdérmica e percutânea evitam o metabolismo hepático e não interferem no sistema renina-angiotensina sendo indicadas principalmente nas pacientes com antecedentes de tromboembolismo e nas hipertensas.

Os estrógenos sintéticos são constituídos principalmente pelo etinilestradiol, mestranol, quimestrol e dietilestilbestrol. O etinilestraiol é o utilizado nos contraceptivos orais, sendo mais potente que o estradiol. Provavelmente, a sua potência elevada e pronunciado efeito sobre o metabolismo hepático deve-se à sua demorada transformação em metabólitos inativos sendo eliminado nas fezes e urina como sulfato e glicuronídios.

A progesterona pode ser utilizada pelas vias oral, intramuscular transdérmica e através da mucosa vaginal. A forma micronizada, ao contrário do que ocorre com a progesterona pura, é bem absorvida e torna-se uma alternativa ao uso de progesterona. A metabolização da progesterona origina vários produtos sendo os principais a pregnanediona, pregnenolona e pregnanediol.

Os hormônios sintéticos apresentam ação semelhante à da progesterona podendo ser obtidos a partir da testosterona ou da própria progesterona. Possuem indicação em diversos setores de endocrinologia ginecológica, como reposição hormonal, indução de amenorréia, tratamento de hiperplasia ou câncer de endométrio, contracepção, tratamento de hiperandrogenismo. Os pregnamos são derivados da 17-hidroxiprogesterona, produto de baixa potência que permite a síntese de caproato de 17-hidroxiprogesterona e do acetato de hidroxiprogesterona, sendo que este último origina quatro importantes hormônios progestacionais, como acetato de clomardinona, ciproterona, acetato de medroxiprogesterona e 
megestrol. O acetato de medroxiprogesterona é utilizado no tratamento de reposição hormonal. O acetato de ciproterona é utilizado puro no tratamento de hiperandrogenismo grave e associado ao estrogênio na pílula anticoncepcional e em reposição hormonal no climatério. $\mathrm{O}$ acetato de clomardinona apresenta efeito antiestrogênico e já foi utilizado como anticoncepcional. O caproato de hidroxiprogesterona, em uso parenteral semanalmente, mantém níveis adequados de progesterona nas pacientes grávidas que tiveram corpo lúteo removido. Acetato de medroxiprogeterona e acetato de ciproterona são os mais utilizados clinicamente 2 .

A tibolona é um progestogênio derivado do noretinodrel com efeitos de estrógeno, progestínico e androgênio usado como alternativa para prevenção primária de osteoporose e efeito dos sintomas da menopausa. É largamente usada há duas décadas na Europa enquanto nos Estados Unidos seu uso ainda não foi licenciado. É usado continuamente, por via oral, na dose de $2,5 \mathrm{mg} /$ dia. A maioria das pacientes permanece em amenorréia, sendo que $10 \%$ apresentam sangramento uterino acíclico.

Quando a TRH usa estrógenos associados aos progestínicos ela é denominada de "combinada". A combinação pode variar entre contínua e seqüencial ou cíclica. A forma mais popular de esquema contínuo combinado é o uso de $0,625 \mathrm{mg}$ de estrógenos conjugados com 2,5mg de medróxiprogesterona. Estrogênio cíclico ou contínuo e progestogênio cíclico são utilizados na perimenopausa quando existe sintomatologia estrogênio-dependente, assim como nos primeiros anos da pós-menopausa. Nos Estados Unidos, dá-se preferência ao esquema com estrógeno cíclico (25 dias por mês de estrogênio conjugado, 0,625mg) e progestínico cíclico (12 a 14 últimos dias de medroxiprogesterona, 5mg). Na Europa, dá-se preferência ao uso contínuo do estrógeno (0,625mg de estrogênio conjugado) com progestínico cíclico (12 a 14 dias do calendário mensal, no início ou final do mês, na dose de $5 \mathrm{mg}$ de medroxiprogesterona). O esquema que utiliza estrogênio isolado, cíclico ou contínuo, é recomendado para mulheres histerectomizadas. O esquema cíclico, caracterizado por 21 dias de reposição hormonal contínua com pausa de sete dias, tem por intuito reduzir a mastalgia. Os estrógenos mais utilizados são: estrogênio conjugado, 0,625$1,25 \mathrm{mg} /$ dia; valerato de estradiol, 1-2mg/dia; estradiol micronizado, 1-2mg/dia; estriol, 2-6mg/ dia. Todos por via oral. Pela via parenteral são utilizados: estradiol percutâneo, 1,5-3mg/dia; estradiol transdérmico, 25-50-100mg/dia; estradiol implante, 25-50-100mg cada 4-6 meses; benzoato de estradiol, $5 \mathrm{mg} / \mathrm{semana}$; hexaidrobenzoato de estradiol, $5 \mathrm{mg}$ a cada três semanas; valerianato de estradiol, $100 \mathrm{mg}$ quinzenais 3 .

\section{Década de 70: evidências de relação entre reposição hormonal com estrógenos e o carcinoma do endométrio}

Entre 1962 e 1973, o mercado farmacêutico norte-americano quadruplicou as vendas de estrógenos usados para reposição hormonal, num período que coincidiu com o aumento do espectro de indicações para o uso de estrógenos após a menopausa e o aumento da incidência de carcinoma do endométrio nesta faixa etária. Ao mesmo tempo, diversas evidências experimentais e epidemiológicas relacionavam os estrógenos ao desenvolvimento do câncer endometrial. Desta forma, foi de grande impacto a publicação no mesmo fascículo de dezembro de 1975, no conceituado periódico New England Journal of Medicine, de dois estudos caso-controle 4,5 que avaliavam a hipótese de que o aumento na incidência do carcinoma do endométrio estava relacionado ao aumento do uso da terapia de reposição hormonal.

Ziel \& Finkle ${ }^{4}$ compararam o histórico de 94 pacientes com diagnóstico de carcinoma de endométrio com 188 controles não histerectomizados obtidos da mesma população de Los Angeles, Estados Unidos. Mulheres que usavam terapia de reposição hormonal com estrógenos conjugados apresentavam um odds ratio de 7,6 (IC95\%: 4,3-13,8) para receber o diagnóstico de câncer endometrial quando comparadas com mulheres que não usavam. Mesmo entre mulheres em uso de estrógenos pelo período mais curto, entre 1 e 5 anos, o odds ratio estimado foi de 5,6. De modo semelhante, Smith et al. 5 compararam 317 pacientes com diagnóstico de adenocarcinoma de endométrio com o mesmo número de mulheres diagnosticadas como portadoras de outras neoplasias ginecológicas da mesma população de Seattle, Estados Unidos. As mulheres expostas ao estrogênio apresentaram um odds ratio de 4,5 (IC95\%: 3,1-6,5) para desenvolver câncer de endométrio. De modo interessante, o risco de mulheres com outros fatores bem estabelecidos para câncer do endométrio, como obesidade e hipertensão, não era afetado pelo histórico de TRH. O achado de que a TRH aumenta o risco de câncer de endométrio apenas em mulheres não obesas foi posteriormente confirmado por grandes estudos prospectivos 6 . A explicação mais plausível para tal observação é que mulheres obesas já apresentam níveis de estrógenos endógenos tão altos - e suficientes para 
carcinogênese - que a influência de estrógenos exógenos é pequena.

No entanto, a relação causal entre o aumento da prescrição de estrógeno exógeno e o aumento na incidência de câncer endometrial não foi tão prontamente aceita pela comunidade científica. Diversas questões foram levantadas com relação a possíveis vieses na interpretação de estudos caso-controle, em particular, a possibilidade de que o diagnóstico de câncer do endométrio em usuárias de TRH poderia refletir apenas um acompanhamento ginecológico mais próximo nessas pacientes, ou mesmo o fato de que estrógenos podem promover sangramento genital chamando atenção para uma neoplasia que, em não usuárias de TRH, permaneceria assintomática 7 . No entanto, por algum tempo, os defensores e opositores da potencial associação entre TRH e câncer de endométrio basearam seus argumentos em dados de estudos caso-controle ${ }^{8}$.

Finalmente, Jick et al. ${ }^{9}$ relataram resultados de estudo de coorte retrospectivo em 1979 que acompanhou uma população de membros de uma cooperativa de assistência médica da área hospitalar de Seattle. Uma primeira observação importante foi queda nacional na incidência de carcinoma endometrial que ocorreu em paralelo com a queda nas prescrições de TRH, em todo o país e na população em estudo, em parte devido a grande exposição na mídia das dúvidas em relação à segurança de seu uso. Na população estudada, a proporção de mulheres entre 50 e 64 anos em uso de TRH cai de 17,5\% (2.795 mulheres) em 1975 para 9\% (1.558 mulheres) em 1979. No período de estudo 65 mulheres receberam o diagnóstico de carcinoma do endométrio. Entre todas as pacientes que desenvolveram carcinoma de endométrio, $75 \%$ eram usuárias correntes de estrogênio por pelo menos seis meses, $15 \%$ utilizaram estrogênio apenas no passado, por pelo menos um ano, e 10\% nunca utilizaram a droga. Calculou-se, portanto, que a incidência de câncer de endométrio entre usuários correntes de estrogênio era de $1 \%$ a $3 \%$ por ano, enquanto a incidência entre os não usuários era de $0,1 \%$ por ano. Uma descrição microscópica do útero retirado após cirurgia identificou invasão miometrial em $43 \%$ das pacientes. Destas, $61 \%$ eram usuárias de estrogênio enquanto $86 \%$ dos casos sem invasão miometrial eram usuários de estrogênio.

Os achados acima serviram de suporte à hipótese de que usuárias de estrogênio desenvolvem neoplasias com tendência menos invasiva quando comparadas com os pacientes portadores da doença que não usam TRH. Sobre esta questão, Shapiro et al. 10 realizaram um novo estudo de caso-controle visando avaliar o risco de neoplasia avançada em pacientes em uso de TRH e os efeitos da interrupção do tratamento. Os casos e controles foram obtidos a partir de hospitais localizados em Boston, Philadelphia, Baltimore, Tucson, Nova York, Kansas, San Francisco e Londres. O grupo controle foi composto por 792 pacientes originários dos setores de ortopedia e cirurgia. O grupo caso foi composto por 425 pacientes com diagnóstico de câncer do endométrio e dividido em duas categorias: 340 com doença localizada (estágios I e II) e 57 com doença extra-uterina (estágios III e IV). Entre os pacientes que desenvolveram adenocarcinoma, $31 \%$ estavam em uso de estrogênio por no mínimo dois anos ou mais, até a data da pesquisa. No grupo controle, $15 \%$ apresentava o mesmo histórico. O risco relativo para o carcinoma do endométrio foi de 2,9 nas usuárias de TRH por 1 a 4 anos; 5,6 em usuárias por 5 a 9 anos e 10 nas usuárias por 10 ou mais anos. Em contraste com avaliações em amostras menores prévias, a tendência para desenvolver câncer de endométrio permaneceu elevada após a descontinuação do tratamento com estrogênio mesmo em intervalos de até 10 anos de interrupção.

Em contraste com sugestões de trabalhos anteriores, Shapiro et al. 10 observaram que a TRH aumentava o risco tanto de câncer localizado quanto avançado. Usuárias de TRH apresentaram um risco relativo de 3,1 (IC95\%: 1,5-6,4) para receber diagnóstico de neoplasia já no estágio extra-uterino. Na discussão deste trabalho, os autores chamaram atenção para dados recentes à época sobre o potencial efeito de progestínicos na oposição ao risco de câncer endometrial usados nos contraceptivos orais (abaixo). Em breve, esquemas de TRH usando combinações de estrógenos e progesterona seriam avaliados como alternativas mais seguras.

\section{Décadas de 80 e 90: a combinação com progesterona antagoniza os efeitos dos estrógenos exógenos para risco de carcinoma do endométrio}

Em 1980, Weiss \& Sayvetz ${ }^{11}$ avaliaram a relação entre desenvolvimento de câncer de endométrio e uso de contraceptivos orais. Diversos estudos prévios já haviam demonstrado que, entre mulheres jovens portadoras de câncer de endométrio e com antecedentes de uso de contraceptivos orais, a maioria utilizava um esquema seqüencial baseado na formulação comercial com alta dose de estrógenos diária $(0,1 \mathrm{mg}$ de etinil estradiol e 25mg de dimetil estrona) combinados com fraco progestínico (25mg dimetilsterona). Neste estudo caso-controle, mulheres brancas entre 35 
e 49 anos residentes no Estado de Washington, Estados Unidos, com diagnóstico de câncer de endométrio entre janeiro de 1975 e dezembro de 1977, foram comparadas com 395 controles pareados para etnia, residência e idade. $\mathrm{O}$ odds $\mathrm{ra}$ tio para câncer endometrial entre as usuárias do esquema seqüencial com altas doses de estrógenos foi de 2,2 (IC95\%: 0,6-7,3) vezes maior que aquele entre os componentes do grupo controle. Para contraceptivos combinados com doses mais altas de progestínicos, verificou-se que o grupo caso apresentava uma menor freqüência de uso, estimando-se que o odds ratio de câncer de endométrio entre os usuários representava $50 \%$ do risco entre os não usuários (odds ratio igual a 0,5; IC95\%: 0,1-1,0). Além disso, verificou-se também que o efeito protetor dos contraceptivos orais combinados sobre o endométrio pode ser influenciado por história prévia de uso de reposição estrogênica. Tal efeito protetor apresentou-se apenas entre as mulheres que não utilizavam reposição estrogênica ou que utilizavam apenas por dois anos ou menos. Por fim, os autores sugeriram que uso de contraceptivos orais combinados diminui o risco de desenvolvimento de carcinoma endometrial 11. A observação do efeito protetor de progestínicos exógenos combinados nos esquemas de contracepção oral serviu de base para adição de progesterona nas terapias de reposição hormonal para mulheres menopausadas.

No início da década de 90, alguns estudos já haviam comprovado o efeito antiproliferativo e de diferenciação glandular da progesterona sobre o endométrio quando utilizada seqüencialmente com estrógenos. Uma coorte sueca de 23 mil pacientes sugeriu também os efeitos de regressão da hiperplasia endometrial induzida por estrógenos e proteção maior quando os progestínicos são utilizados entre 10 a 13 dias por mês. Neste estudo, o uso de estrógenos sem oposição por progestínico aumentou o risco de carcinoma do endométrio em 2,2 (IC95\%: 1,2-4,4) vezes, enquanto a combinação com progesterona exibia risco similar aos controles: 0,9 (IC95\%: 0,4-2,0) 12. Em 1991, Voight et al. 13 publicaram um estudo caso-controle avaliando o efeito de progestínicos exógenos sobre a incidência de câncer de endométrio entre usuários de estrógenos para reposição hormonal. O grupo caso foi composto por 158 mulheres entre 40 e 64 anos com diagnóstico de câncer de endométrio identificados nos anos de 1985 a 1987 residentes do Estado de Washington. O grupo controle foi composto por 182 mulheres selecionadas por randomização dos números telefônicos. Entre as mulheres que utilizavam estrógenos exógenos, mas nunca utilizavam progestínicos, o odds ratio de câncer de endométrio foi de 5,7 (IC95\%: 2,5-12,8) quando comparadas às mulheres que nunca utilizaram qualquer tipo de reposição hormonal. Quanto às usuárias de estrógenos e progestínicos, não houve um aumento significativo no risco de câncer endometrial quando comparadas com as não usuárias de qualquer tipo de reposição hormonal. Dividindo-se as usuárias de reposição estrogênica e progestínica quanto ao número de dias, por mês, observou-se um odds ratio de 2,4 (IC95\%: $0,6-9,3)$ entre as mulheres que utilizavam progestínicos por menos de dez dias por mês. Entre aquelas que utilizavam progestínicos por mais de dez dias por mês, o odds ratio apresentou-se 1,1 (IC95\%: 0,4-3,0). Tais resultados sugeriram que o efeito protetor dos progestínicos é maior quando são utilizados por mais de dez dias por mês.

Em 1996, foram divulgados os resultados do ensaio clínico Postmenopausal Estrogen/ Progestin Intervention (PEPI) Trial sobre os efeitos da TRH sobre a morfologia endometrial 14 . O PEPI Trial consistiu-se em um estudo de coorte, multicêntrico, randomizado duplo-cego que acompanhou 875 mulheres durante três anos avaliando efeito de diversos tipos de reposição hormonal sobre os fatores de risco de cardiopatia como níveis de HDL, fibrinogênio, insulina e tensão arterial. Porém, o estudo forneceu também uma oportunidade para avaliar os efeitos da reposição hormonal sobre a histologia do endométrio. Neste, 596 mulheres entre 45 e 64 anos, não histerectomizadas, foram divididas em três grupos: grupo placebo, usuárias de estrógenos e usuárias de estrógeno e progestínicos. As usuárias de estrógenos usavam estrógeno conjugado $(0,625 \mathrm{mg} / \mathrm{dia})$ enquanto os esquemas combinados incluíam essa dose de estrógenos com acetato de medroxiprogesterona $(10 \mathrm{mg} / \mathrm{dia}$ ou 2,5mg/dia) nos primeiros 12 dias do mês; estrógeno conjugado e progesterona micronizada (200mg/dia) também ao longo dos primeiros 12 dias do mês. Todos os regimes eram cíclicos e duravam 28 dias. Todos os medicamentos foram usados durante três anos. A histologia endometrial foi avaliada a partir de biópsias, que classificaram o endométrio como normal, sob hiperplasia simples (cística), hiperplasia complexa (adenomatosa), hiperplasia atípica e adenocarcinoma. Um total de 506 mulheres (85\%) apresentaram resultados normais ao longo de todas a biópsias de acompanhamento. Entre as mulheres que utilizaram apenas estrógeno conjugado, 74 de 119 (62,2\%) desenvolveram algum tipo de hiperplasia endometrial e 41 de 119 $(34,4 \%)$ desenvolveram hiperplasia complexa ou atípica. Entre as usuárias de estrógenos e progestínicos, observaram-se dez casos de hiperplasia simples, dois de hiperplasia complexa e um caso 
de hiperplasia atípica. A incidência de hiperplasia entre usuárias de estrógenos sem oposição de progestínicos e controles foi de $28 \%$ vs. $1 \%$ para hiperplasia simples, $23 \%$ vs. $1 \%$ para complexa e $1 \%$ vs. $0 \%$ para atípica. Não houve diferença nos resultados das biópsias entre o grupo placebo e o grupo sob reposição estrogênica e progestínica. De fato, o monitoramento do endométrio visava detectar principalmente as hiperplasias complexa e atípica, consideradas como prognóstico de malignidade, assim como o próprio adenocarcinoma de endométrio. Como conclusão, o PEPI Trial orientou os clínicos a avaliarem a possibilidade de adicionar progestínicos, cíclica ou continuamente, devido ao seu efeito protetor sobre o endométrio. Segundo os achados do estudo, numa dosagem de 0,625mg, a administração diária de estrógeno conjugado aumenta a incidência de hiperplasia endometrial, enquanto a combinação de estrógeno conjugado com acetato de medroxiprogesterona, cíclica ou continuamente, protege o endométrio do efeito proliferativo do estrógeno.

Woodruff \& Pickar 15, em 1994, realizaram também um estudo prospectivo randomizado duplo-cego ao longo de um ano que visava comparar a incidência de hiperplasia endometrial entre os diversos regimes de reposição hormonal incluindo tanto esquemas combinados cíclicos quanto contínuos. As doses de medroxiprogesterona foram $2,5 \mathrm{mg}$ ou $5 \mathrm{mg}$ diariamente, ou $5 \mathrm{mg}$ ou 10mg por 14 dias de cada ciclo de 28 dias. A incidência de hiperplasia do endométrio foi de $20 \%$ nas mulheres em uso de estrógenos conjugados sem oposição e menor que $1 \%$ nos quatro esquemas de TRH combinada. Os esquemas cíclicos com maiores doses de progestínicos não tiveram nenhum caso de hiperplasia detectado. Apesar da sugestão de que a dose de $2,5 \mathrm{mg}$ de medroxiprogesterona poderia ter o mesmo efeito protetor que altas doses, Comerci Jr. et al. 16, em 1997, relataram resultados adversos de um regime de reposição hormonal contínuo baseado em baixas doses de progestínicos. Foram relatados oito casos de mulheres de Nova York, Estados Unidos, que desenvolveram câncer de endométrio enquanto submetiam-se a tal regime de reposição hormonal. De acordo com os pesquisadores, a reposição contínua de estrógeno e progesterona aumentaria significativamente a condescendência dos pacientes em relação ao tratamento, pois eliminaria o retorno das menstruações, ganho de peso e sintomas depressivos. De fato, o American College of Obstetricians and Gynecologists (ACOG) recomendava que fosse reconsiderada a dose de medroxiprogesterona de $10 \mathrm{mg}$ para $2,5 \mathrm{mg}$ com base na observação que tal regime era eficaz na prevenção de fraturas osteoporóticas e não prejudicava o efeito dos estrógenos sobre o aumento sérico de lipoproteína de alta densidade (HDL) sérica. No entanto, tal estudo relatou oito casos de TRH combinada usando 2,5mg de medroxiprogesterona e questionou o efeito protetor deste esquema.

Um outro esquema proposto para reduzir os efeitos da TRH sobre o endométrio foi o uso de baixas doses de estrógenos 0,3mg/dia sem oposição de progestínicos. Num estudo caso-controle realizado no Estado de Washington, 832 casos e 1.114 controles foram avaliados e o uso de TRH com estrógenos em baixas doses foi associado a um odds ratio de 5,2 (IC95\%: 2,3-13,0) para receber diagnóstico de câncer do endométrio. Tal risco subia para 9,2 (IC95\%: 2,9-29,0) quando avaliadas apenas mulheres em uso deste esquema por mais de oito anos 17. Desta forma, a redução apenas no componente estrogênico não reduz o efeito carcinogênico sobre o endométrio.

Por fim, em 1998, Ziel et al. 18 realizaram um estudo ecológico que relacionou a incidência de carcinoma endometrial com a prescrição de hormônios numa população assistida por plano de saúde específico de Los Angeles, entre 1971 e 1993. A incidência da doença cresceu entre 1971 e 1975 em paralelo com o aumento das prescrições de estrógenos para reposição hormonal. A taxa de câncer de endométrio cai de 97 para 38 casos por 100 mil mulheres entre 1975 e 1992. Tal queda é explicada parcialmente pela acentuada queda nas prescrições de estrógenos para TRH entre 1975 e 1977 como efeito da divulgação na mídia dos primeiros relatos que associaram TRH com aumento do risco de câncer. No entanto, as prescrições de estrógenos voltam a delinear uma curva ascendente a partir de 1978 e, com o aumento das prescrições de progesterona a partir de 1983, a maior parte do declínio na incidência do câncer do endométrio na população é explicável pelos efeitos da TRH combinada. As prescrições de medroxiprogesterona aumentaram de 2,2 comprimidos por indivíduo, em 1983, para 27,3 comprimidos por indivíduo em 1993. A menor incidência de câncer de endométrio ocorreu entre 1991 e 1993. Curiosamente, neste mesmo período, observou-se a maior taxa de prescrição de estrógenos. Porém, neste mesmo período, a taxa de prescrição de medroxiprogesterona apresentava-se elevada e constante, o que ratifica o efeito protetor dos progestínicos sobre o endométrio. 


\section{Questões atuais: risco total de câncer e efeitos adversos nos esquemas de terapia de reposição hormonal combinada}

A TRH chegou ao ano 2000 com algumas certezas, que incluíam o efeito protetor da combinação com progestínicos sobre o endométrio, e várias dúvidas referentes a até que ponto benefícios e efeitos adversos podiam ser quantificados para guiar a decisão de usar ou não usar cada esquema. Num grande estudo denominado WHI (Women Health Iniatiative), foi desenhado um ensaio clínico controlado randomizado com mais de 160 mil mulheres entre 50 e 79 anos acompanhadas entre 1993 e 1998. O estudo foi interrompido precocemente com 5,2 anos de média de seguimento porque os efeitos deletérios da TRH combinada superaram seus benefícios. Neste estudo, 8.506 mulheres passaram a usar $0,625 \mathrm{mg}$ de estrógenos conjugados com 2,5mg de medroxiprogesterona e 8.102 mulheres passaram a usar placebo. Mulheres em uso de TRH combinada foram beneficiadas por um menor risco de fraturas (odds ratio = 0,76; IC95\%: 0,69-0,85), mas apresentaram maior risco de apresentar doença cardiovascular (odds ratio = 1,29; IC95\%: 1,02-1,63), acidente vascular cerebral (odds ratio $=1,41$; IC95\%: 1,07-1,85) e tromboembolismo pulmonar (odds ratio = 2,13; IC95\%: 1,39-3,25). Quanto ao risco de câncer, a TRH combinada manteve o mesmo risco de carcinoma do endométrio que os controles (odds ratio = 0,83; IC95\%: 0,47-1,47), foi protetora para carcinoma do cólon e reto (odds ratio= 0,63; IC95\%: 0,43-0,92) e aumentou o risco de carcinoma da mama (odds ratio = 1,26; IC95\%: 1,00-1,63). O estudo foi interrompido pelo maior risco de câncer de mama e de eventos cardiovasculares. Não houve aumento estatisticamente significante de câncer total ou da mortalidade geral, no entanto, a incidência geral de eventos adversos foi 1,15 vezes (IC95\%: 1,03-1,28) maior que nos controles 19 .

Esta observação seguiu-se ao relato prévio de que a TRH combinada aumenta o risco de eventos cardiovasculares em mulheres com cardiopatia prévia 20 . Já no estudo WHI, apenas 7,7\% das mulheres tinham cardiopatia prévia e, portanto, a população estudada representava os efeitos do TRH predominantemente em indivíduos saudáveis. Em conjunto, os resultados contra-indicam o uso de esquemas combinados para prevenção primária e secundária de doença cardíaca isquêmica em mulheres nesta faixa etária. Nestes trabalhos, o risco de eventos cardiovasculares é maior no primeiro ano de uso da reposição hormonal. Uma série de estudos observacionais prévios que sugeriram um efeito benéfico da TRH combinada sobre a doença cardiovascular deve ser reavaliada hoje como estudos que apresentam fortes vieses 21 . Na realidade, alguns autores reavaliaram seus dados e detectaram um aumento precoce em eventos cardiovasculares no subgrupo de pacientes já cardiopatas após início do uso de TRH 19. Uma justificativa para o maior risco cardiovascular no primeiro ano de uso seria uma tendência pró-trombótica causada por estrógenos que pode acelerar a progressão da doença cardiovascular, enquanto os benefícios sobre o metabolismo lipídico podem equilibrar o risco cardiovascular a longo prazo.

Portanto, um problema emergente era a associação potencial entre TRH combinada e o câncer de mama após o resultado do estudo WHI e de uma análise de 51 estudos observacionais que estimou um aumento de $53 \%$ de risco para câncer de mama em mulheres que usavam TRH combinada ou progestínicos exógenos apenas 22. Um grande estudo caso-controle de base populacional foi desenhado para avaliar o efeito de diferentes esquemas de TRH a longo prazo na incidência do câncer de mama. O estudo foi conduzido em Seattle e identificou 975 mulheres entre 65 e 79 anos que receberam diagnóstico de câncer entre 1997 e 1999. A TRH combinada estava relacionada à maior incidência de carcinoma da mama tanto lobular (odds ratio $=1,5$; IC95\%: $1,1-2,0$ ) quanto ductal (odds ratio $=2,7$; IC95\%: $1,7-4,3$ ) sendo maior nos subgrupos com uso de longo prazo e não diferindo quanto ao esquema contínuo ou cíclico. De modo interessante, a TRH com estrógeno apenas não aumentou o risco de câncer de mama quando comparado com mulheres que jamais usaram TRH 23. A relação entre estrógenos exógenos e o carcinoma da mama, entretanto, já fora sugerida em outros estudos 22 .

Uma grande coorte que arrolou mais de um milhão de mulheres, um quarto da população feminina inglesa entre 50 e 64 anos durante os anos de 1996 e 2001, foi desenhada para comparar os efeitos de diferentes esquemas de TRH: combinado contínuo (69 mil mulheres), combinado cíclico (145 mil), tibolona (28 mil) e estrógeno puro (14 mil); que foram comparados com 395 mil mulheres que jamais usaram TRH. Quanto ao risco de câncer do endométrio, o esquema contínuo combinado mostrou-se protetor (odds ratio $=0,71$; IC95\%: 0,56-0,90) enquanto o esquema combinado cíclico não teve efeito (odds ratio $=1,05$; IC95\%: 0,91-1,22) e dois esquemas aumentaram o risco da doença: tibolona (odds ratio = 1,79; IC95\%: 1,43-2,25) e estrógenos apenas (odds ratio = 1,45; IC95\%: 1,02-2,06). A estratificação quanto ao índice de massa corpórea revelou dados interessantes. A incidência é menor 
em obesas do que em mulheres com sobrepeso que usam terapia contínua combinada, mas é maior nas obesas tratadas com tibolona ou TRH combinada cíclica. No entanto, a tibolona tem um odds ratio maior que mulheres que nunca usaram TRH e a TRH cíclica tem odds ratio menor que os controles. Isto ocorre porque em obesas a incidência de câncer endometrial aumenta mais nas que nunca usaram TRH do que nas que usaram (com exceção das usuárias de tibolona que exibem o maior risco). Portanto, esta relação apenas ilustra o fato de que o excesso de estrógenos endógenos nas obesas torna os efeitos de hormônios exógenos menos dramáticos sobre a incidência do câncer de endométrio 6 .

O principal achado deste estudo, no entanto, foi a observação de que qualquer um dos quatro esquemas de TRH aumenta o risco de câncer de mama quando comparadas às mulheres que jamais fizeram reposição hormonal. Além disso, este aumento é maior nos esquemas combinados. A incidência de câncer (casos por mil mulheres/ano) de endométrio para cada esquema foi de 2,0 (IC95\%: 1,5-2,6) para TRH combinada contínua; 3,0 (IC95\%: 2,5-3,5) para TRH combinada cíclica; 5,7 (IC95\%: 4,8-7,6) para tibolona; 4,9 (IC95\%: 3,5-7,5) para estrógeno apenas; 3,0 (IC95\%: 2,8-3,2) para mulheres que jamais usaram TRH. A incidência de câncer de mama (casos por mil mulheres) para cada esquema foi de: 29,3 (IC95\%: 27,5-31,2) para TRH combinada contínua; 27,9 (IC95\%: 26,1-29,8) para TRH combinada cíclica; 19,9 (IC95\%: 17,9-22,5) para tibolona; 18,0 (IC95\%: 17,1-20,0) para estrógeno apenas; 13,6 (IC95\%: 13,2-14,1) para mulheres que jamais usaram TRH. A conclusão do estudo é clara: a TRH combinada reduz o risco de carcinoma do endométrio, mas aumenta o risco do câncer de mama e, como o câncer de mama é muito mais comum que o endometrial, isso significa que a TRH combinada está associada a um risco total de câncer maior. O risco geral de câncer é maior que o observado nas mulheres que jamais usaram reposição hormonal e, de modo surpreendente, também é maior quando comparado ao grupo de mulheres que usam apenas estrógenos. Neste estudo, a incidência total (endométrio + mama) na população estudada obedeceu a seguinte ordem decrescente: TRH contínua combinada (31,3 casos/mil/ano), TRH contínua cíclica $(30,9)$, tibolona $(25,6)$, estrógeno apenas $(22,9)$ e nenhuma TRH $(16,6) 6$.

A questão imposta, portanto, é de qual a aplicação da reposição combinada com progesterona à luz dos dados atuais. Como justificar seu uso para prevenção do risco de carcinoma endométrio se os dados mais recentes chamam atenção para o maior risco de doença cardiovascular e câncer total? O consenso da Sociedade Brasileira do Climatério constata que não há justificativa para indicação de TRH para prevenção primária ou secundária de doença cardiovascular ressaltando o papel importante que a reposição hormonal pode ter especialmente quando indicada para atrofia genital e osteoporose. Entre as críticas levantadas às recentes publicações aqui relatadas, o consenso chama atenção para o fato de que o risco cardiovascular não foi avaliado apenas em mulheres na perimenopausa (50 a 55 anos). As considerações específicas de quando usar ou não a combinação com progesterona não são claras no consenso 24 . De forma semelhante, os achados de eventos adversos são minimizados no manual da Federação Brasileira das Associações de Ginecologia e Obstetrícia 25 . Ressalte-se, porém, que ambas publicações são anteriores aos achados mais recentes referentes ao aumento da incidência de câncer de mama com esquemas combinados 6 .

\section{Conclusão}

Desta forma, à luz dos conhecimentos atuais, o efeito protetor que a combinação de progestínicos apresenta sobre o risco de carcinoma do endométrio não parece contrabalançar uma série de outros efeitos adversos da TRH combinada, incluindo o risco de outros tumores malignos mais freqüentes. Portanto, o uso de TRH combinada não é justificado para a população feminina na pós-menopausa para prevenção primária de câncer do endométrio. Além disso, não há literatura científica disponível para definir doses seguras de qualquer esquema de TRH. 


\section{Resumo}

A reposição hormonal para alivio dos sintomas menopausais é amplamente utilizada em todo o mundo. A evolução do conhecimento sobre os riscos deste tratamento sempre foi mais lenta do que sua aplicação na prática clínica. Na década de 70, um aumento de incidência de câncer do endométrio ocorreu nos países desenvolvidos sendo que a terapia de estrógenos exógenos na menopausa foi o principal fator relacionado. Nas décadas de 80 e 90, a combinação entre estrógenos e progestínicos passou a ser largamente utilizada com base na premissa de que apresentava efeitos benéficos sobre os sistemas cardiovascular e osteoarticular, sem aumento no risco de câncer uterino. Entretanto, relatos recentes novamente questionam a segurança da reposição hormonal e, desta vez, apontam para o risco maior de câncer total e doença cardiovascular nos esquemas combinados. Concluímos neste trabalho que os riscos recentemente relacionados à terapia combinada têm grande potencial de impacto na Saúde Pública, e este esquema não é indicado para proteção do risco de carcinoma endometrial uma vez que seus riscos superam os benefícios.

Terapia de Reposição Hormonal; Neoplasias do Endométrio; Saúde da Mulher

\section{Referências}

1. Amant F, Moerman P, Neven P, Timmerman D, van Limbergen E, Vergote I. Endometrial cancer. Lancet 2005; 366:491-505.

2. Viana LC, Martins M, Geber S. Climatério. In: Alves J, organizador. Ginecologia. Rio de Janeiro: Medsi Editora; 1998. p. 504-8.

3. Federação Brasileira das Associações de Ginecologia e Obstetrícia. Tratado de ginecologia. Rio de Janeiro: Editora Revinter; 2001.

4. Ziel HK, Finkle WD. Increased risk of endometrial carcinoma among users of conjugated estrogens. N Engl J Med 1975; 293:1167-70.

5. Smith DC, Prentice R, Thompson DJ, Herrmann WL. Association of exogenous estrogen and endometrial carcinoma. N Engl J Med 1975; 293:1164-7.

6. Beral V, Bull D, Reeves G. Endometrial cancer and hormone-replacement therapy in the Million Women Study. Lancet 2005; 365:1543-51.

\section{Colaboradores}

Ambos os autores participaram da redação do artigo, selecionando e discutindo de forma crítica os principais relatos da literatura sobre o tema.
7. Horwitz RI, Feinstein AR. Alternative analytic methods for case-control studies of estrogens and endometrial cancer. N Engl J Med 1978; 299: 1089-94.

8. Case-control studies of estrogens and endometrial cancer. N Engl J Med 1979; 300:495-7.

9. Jick H, Watkins RN, Hunter JR, Dinan BJ, Madsen S, Rothman KJ, et al. Replacement estrogens and endometrial cancer. N Engl J Med 1979; 300: 218-22.

10. Shapiro S, Kelly JP, Rosenberg L, Kaufman DW, Helmrich SP, Rosenshein LB, et al. Risk of localized and widespread endometrial cancer in relation to recent and discontinued use of conjugated estrogens. N Engl J Med 1985; 313:969-72.

11. Weiss NS, Sayvetz TA. Incidence of endometrial cancer in relation to the use of oral contraceptives. N Engl J Med 1980; 302:551-4. 
12. Persson I, Adami HO, Bergkvist L, Lindgren A, Pettersson B, Hoover R, et al. Risk of endometrial cancer after treatment with oestrogens alone or in conjunction with progestogens: results of a prospective study. BMJ 1989; 298:147-51.

13. Voigt LF, Weiss NS, Chu J, Dailing JR, McKnight B, van Belle G, et al. Progestagen supplementation of exogenous oestrogens and risk of endometrial cancer. Lancet 1991; 338:274-7.

14. Effects of hormone replacement therapy on endometrial histology in postmenopausal women. The Postmenopausal Estrogen/Progestin Interventions (PEPI) Trial. The Writing Group for the PEPI Trial. JAMA 1996; 275:370-5.

15. Woodruff JD, Pickar JH. Incidence of endometrial hyperplasia in postmenopausal women taking conjugated estrogens (Premarin) with medroxyprogesterone acetate or conjugated estrogens alone. The Menopause Study Group. Am J Obstet Gynecol 1994; 170(5 Pt 1):1213-23.

16. Comerci Jr. JT, Fields AL, Runowicz CD, Goldberg GL. Continuous low-dose combined hormone replacement therapy and the risk of endometrial cancer. Gynecol Oncol 1997; 64:425-30.

17. Cushing KL, Weiss NS, Voigt LF, McKnight B, Beresford SA. Risk of endometrial cancer in relation to use of low-dose, unopposed estrogens. Obstet Gynecol 1998; 91:35-9.

18. Ziel HK, Finkle WD, Greenland S. Decline in incidence of endometrial cancer following increase in prescriptions for opposed conjugated estrogens in a prepaid health plan. Gynecol Oncol 1998; 68:253-5.
19. Rossouw JE, Anderson GL, Prentice RL, LaCroix AZ, Koopenberg C, Stefanick ML, et al. Risks and benefits of estrogen plus progestin in healthy postmenopausal women: principal results from the Women's Health Initiative randomized controlled trial. JAMA 2002; 288:321-33.

20. Hulley S, Grady D, Bush T, Furberg C, Herrington D, Riggs B, et al. Randomized trial of estrogen plus progestin for secondary prevention of coronary heart disease in postmenopausal women. Heart and Estrogen/Progestin Replacement Study (HERS) Research Group. JAMA 1998; 280:605-13.

21. Beral V, Banks E, Reeves G. Evidence from randomised trials on the long-term effects of hormone replacement therapy. Lancet 2002; 360:942-4.

22. Breast cancer and hormone replacement therapy: collaborative reanalysis of data from 51 epidemiological studies of 52,705 women with breast cancer and 108,411 women without breast cancer. Collaborative Group on Hormonal Factors in Breast Cancer. Lancet 1997; 350:1047-59.

23. Li CI, Malone KE, Porter PL, Weiss NS, Tang MT, Cushing-Haugen KL, et al. Relationship between long durations and different regimens of hormone therapy and risk of breast cancer. JAMA 2003; 289:3254-63.

24. Fernandes CE. Consenso sobre a terapêutica hormonal na menopausa: controvérsias da terapêutica hormonal $(\mathrm{TH})$ na mulher climatérica. São Paulo: Editora Medcomm; 2004.

25. Fernandes CE, Baracat EC, Lima GR. Manual do climatério. São Paulo: Editora Ponto; 2004.

Recebido em 05/Mai/2006

Aprovado em 09/Mai/2007 